\title{
Cidades biofílicas inteligentes: um estudo sobre diretrizes deste conceito aplicado a cidades médias
}

\author{
Ciudades biofílicas inteligentes: un estudio sobre directrices de \\ este concepto aplicado a ciudades medias
}

\author{
Smart biophilic cities: a study on guidelines of this concept \\ applied to medium-sized cities
}

\author{
Luan da Silva Klebers \\ luan.klebers@hotmail.com \\ Universidade Federal de Santa Maria, UFSM, Santa Maria, RS \\ Luis Guilherme Aita Pippi \\ guiamy@hotmail.com \\ Universidade Federal de Santa Maria, UFSM, Santa Maria, RS
}

\begin{abstract}
Resumo: A temática de cidades inteligentes é tratada como uma forma de compreender o planejamento urbano e traçar novas perspetivas para que, através da plena utilização tecnológica, sejam buscadas melhorias para o cotidiano. No entanto, de forma errônea por muitas vezes, se segrega a 'inteligência' da relação ecossistêmica da sociedade com a natureza. A cidade é vital, dinâmica e democrática, oriunda da relação simbiótica de coexistência dos meios antrópico e natural, exigindo multifuncionalidade e dinâmica de seus usuários. Este trabalho é uma breve reflexão acerca de cidades biofílicas inteligentes analisando cidades médias sob a ótica de cidades resilientes, e sua aplicabilidade conceitual.
\end{abstract}

Palavras-chave: cidades inteligentes; cidades resilientes; planejamento urbano.

Resumen: La temática es tratada como forma de comprender la planificación urbana y trazar nuevas perspectivas para que, a través de la plena utilización tecnológica sea buscada mejoras para el cotidiano. Pero de forma errónea por mucho as veces, se segrega la "inteligencia" de la relación ecosistémica de la sociedad con la naturaleza. La ciudad es vital, dinámica y democrática, es oriunda de la relación simbiótica de coexistencia del medio antrópico y del medio natural, siendo necesaria multifuncionalidad y dinámica de sus usuarios. Este trabajo es una breve reflexión acerca de ciudades biofílicas inteligentes analizando el medio de ciudades medias bajo la óptica de ciudades resilientes y su aplicabilidad conceptual.

Palabras clave: ciudades inteligentes; ciudades resilientes; planeamiento urbano.

Abstract: The issue is presented to understand urban planning and how draw new perspectives so that, through full technological use, improvements are sought for everyday life. However, it fails by segregating the 'smart' concept from society's ecosystem relationship with nature. The city is vital, dynamic and democratic, it comes from the symbiotic relationship of coexistence amid anthropic and natural environments thus requiring multifunctionality and dynamics of users. This paper 
presents a brief reflection about smart biophilic cities, analyzing the medium-sized cities from the perspective of resilient cities, and their conceptual applicability.

Keywords: smart cities; resilient cities; urban planning.

\section{INTRODUÇÃO}

As cidades médias constituem importante temática para a geografia urbana. Exibindo inúmeras possibilidades de estudo, sua conceitualização é de natureza relacional, não possuindo mensurações ontologicamente definidas, uma vez que é resultado de construção intelectual devido a adoção de critérios julgados pertinentes pelos pesquisadores, de acordo com os aspectos contextuais individuais de sua rede urbana. Outrossim, são aspectos importantes, por exemplo, suas funções, extensão, densidade demográfica, crescimento vegetativo, dentre outros. Spósito (2017, p.18), relatam que "as cidades médias e pequenas ocupam um lugar à parte na investigação sobre o urbanismo" - lembrando que não podemos, para estudá-las, "copiar as pesquisas feitas sobre metrópoles", devendo assim as analisarmos em sua totalidade e complexidade de acordo com sua inserção.

O surgimento de novos serviços, principalmente os de logística, transportes, informação, comunicação, educação e turismo, acarretaram em inúmeras transformações sob o ambiente urbano. Em 1980 surgiram as primeiras ideias das cidades médias tornandose atrativas em relação às grandes metrópoles, visto que sua maior capacidade de expansão, com geração de empregos e melhores condições de vida (PONTES, 2005). Esse crescimento, porém, não ocorreu de forma inteligente, deixando assim um legado edificado que precisa ser adaptado.

Há diversas experiências no desenvolvimento de políticas de descentralização territorial, possibilitando assim um acúmulo de informações teóricas sobre o quesito hierárquico das cidades, o que torna particularmente difícil formular definições absolutas, sendo necessário levar em conta inúmeros aspectos ímpares para a tomada de decisões (ANDRADE; SERRA, 2001).

O processo evolutivo das cidades renova-se constantemente, principalmente devido ao surgimento de novas tecnologias. Não só a urbe, mas também o espaço é alterado por "mudanças no padrão tecnológico e produtivo que se fazem acompanhar da emergência de novas formas espaciais, ou da presença de velhas formas espaciais com novos conteúdos" (SILVEIRA, 2003, p.12). Desta forma, pode-se afirmar que nas últimas décadas a inovação tecnológica transformou o pensamento do ser humano através de dispositivos eletrônicos e aplicativos capazes de realizar uma quantia imensurável de tarefas, tornando-se cada vez mais essenciais para nosso bem-estar.

A metamorfose diária da maneira com que se vive impulsiona melhorias de cotidiano, porém muitas cidades acabam por não acompanhar este processo, visto que a demanda por investimentos do poder público e cooperação da sociedade na qual está inserida tornam difícil e lento o processo evolutivo do espaço construído. A sociedade se desenvolve, aumentando proporcionalmente o consumo de recursos naturais, mas nem sempre de um 
modo eficiente e consciente (ADENE, 2010, p.7). “É necessário por isso, a integração de medidas de eficiência energética e de avaliação de sustentabilidade à escala do município numa ação inovadora e abrangente a ser realizada de modo que seja possível continuar a intervir no território, mas compatibilizando as atividades humanas com o meio natural e construído, salvaguardando a componente energética de um modo equitativo e eficaz." (AMADO et al., 2014, p. 23)

Surge então, oriundo desta perspectiva, o conceito de Cidades Biofílicas Inteligentes, cujo objetivo é modular os desafios sociais que a rápida urbanização traz (GIL-GARCIA et al., 2016), minimizando problemas através da utilização de plataformas integradas para adaptar as demandas dos cidadãos às necessidades da cidade (e vice-versa) (ZOMER; MOUSTAID; MEIJER, 2015).

\section{CIDADES BIOFILICAS INTELIGENTES}

De acordo com Cury (2016) a nomenclatura de Cidade Inteligente está totalmente ligada aos conceitos básicos de sustentabilidade, ou seja, prover ambientes onde a construção da sociedade preze pela relação harmoniosa com o meio ambiente, utilizando e reaproveitando de forma consciente e racional os recursos ambientais.

Komninos (2008) pontua que para as cidades inteligentes se concretizarem estão implícitas as dimensões humanas (ecossistêmica), coletiva e artificial. A dimensão humana envolve pessoas com habilidades e aptidões diferentes para assim contribuírem de forma ímpar; a dimensão coletiva diz respeito ao ambiente construído e a inteligência nas instituições de ensino e inovação tecnológica. Por fim, a dimensão artificial se refere aos espaços de comunicação e inovação. Desta forma o andamento da inovação tecnológica estaria sempre ao lado das necessidades comuns.

Já o termo cidade biofílica, conforme Beatley (2010), trata-se de uma cidade imersa à natureza, com abundância de sistemas naturais que sejam visíveis e acessíveis a todos os usuários, isto é, trata-se de uma condição física do espaço em si, porém não somente, envolve o design verde (parques, manchas, espaços livres, vida selvagem urbana, ambientes caminháveis..) porém também faz jus ao imaginário das pessoas, o compromisso emocional e a topofilia que o convívio com a natureza proporciona.

Este pode estar presente nos mais diversos lugares, nas edificações, por exemplo, na forma de telhados verdes, terraços jardins, claraboias, jardins verticais, paredes verdes, dentre outros. Também se aplica ao urbanismo de ruas, quadras, bairros e comunidades, através de calçadas verdes, canteiros centrais, praças, parques de bolso, hortas comunitárias, florestas urbanas, ... promovendo a conectividade entre os elementos da paisagem urbana. Ainda de acordo com Beatley, o conceito de biofilia aplicado à uma cidade está altamente vinculado a sua capacidade de manter a infraestrutura verde e azul, ou seja, aos vários tipos de integração entre a natureza (árvores, vegetação rasteiras, parques, praças, córregos, lagos, florestas, animais de estimação, animais nativos...) e o meio antrópico (pavimentação, edificações, transporte coletivo ...). 
Uma cidade biofílica nada mais é que uma cidade verde, com abundância de recursos naturais e que sejam acessíveis para todos de forma democrática, possibilitando a vida selvagem e a caminhabilidade tomarem rumos paralelos. Entretanto, em mesma intensidade e altamente ligado ao conceito de cidades biofílicas tem-se o conceito de cidades resilientes. A resiliência é a capacidade de um sistema absorver impactos e manter suas funções ou propósitos, isto é, sobreviver ou persistir em um ambiente com variações, incertezas. "As cidades são sistemas socioecológicos, com complexos ciclos adaptativos" (HERZOG, 2013, p. 79).

Não se deve esquecer que as questões relacionadas à infraestrutura verde não se restringem apenas à vegetação, mas sim vinculam todos os conceitos de produção de energia, de qualidade da água, de encontrar modos seguros de conviver com os detritos e reivindicar locais pós-industriais, protegendo contra inundações e revertendo a perda da biodiversidade em variadas escalas (PELLEGRINO; MOURA, 2017).

No entanto, a busca pela otimização ou eficiência de nossos sistemas básicos elimina a diversidade, ou a multifuncionalidade de alternativas, sendo exatamente esta flexibilidade o que manteria as diretrizes de resiliência. Herzog (2013) traz um exemplo reflexivo acerca do controle de inundações, onde afirma que atualmente é considerado eficiente drenar as águas da chuva o mais rápido possível através de uma infraestrutura cinza tradicional, considerada monofuncional. Para isto rios são retificados e canalizados, construindo 'piscinões' de concreto em suas margens. Porém existem outras opções, como a drenagem das águas das chuvas pelo aumento de áreas de infiltração, logo, ao invés de impermeabilizar completamente os canais gerando fluxos contínuos e rápidos, a abordagem de um sistema multifuncional e biofílico se tornaria mais eficiente, além de flexibilizar as oportunidades de espaços de lazer e recreação da paisagem.

Em concordância, Bélanger (2009 p. 85 apud PELLEGRINO 2017, p. 7), afirma que a infraestrutura convencional tem demonstrado capacidade limitada frente aos problemas da urbanização em massa, uma vez que "historicamente a abordagem monofuncional no projeto de infraestruturas tipicamente tem segregado água, lixo, transporte, alimentos e energia em departamentos separados sem nenhuma relação", lembrando que a utilização deste por longos períodos de tempo pode ser perigoso.

Nesse norte, devido à necessidade de melhorar a qualidade de vida e dos espaços urbanizados, deve-se ser desenvolvido e aprimorado a gestão destes locais e a maneira como a cidade os influencia (BLOOMBERG; CROSBY; BRYSON, 2014) ou seja, por meio de gestões efetivas que se baseiam em políticas urbanas integradas (ROSSETO, 2003) mas também que, utilizando das TIC (Tecnologia de Informação e Comunicação), forneçam meios de monitoramento dos serviços de infraestrutura urbana, visto que trata-se do contexto urbano do futuro (NAM; PARDO, 2011)

Lemos (2013) fala que o termo digital por muito tempo fora entendido como o acesso a computadores de forma intuitiva e a implantação de conexões de internet no espaço público e urbano. $\mathrm{O}$ conceito de inteligente diz respeito a processos informatizados suscetíveis a modificações de contexto, trabalhando com grandes volumes de dados (big data), redes em nuvens, comunicação autônoma entre elementos (internet), de forma que inteligente seja 
sinônimo de uma cidade na qual tudo é sensível ao ambiente, ou seja, produz, consome e distribui grandes números de informações em tempo real, processamento este que serve como referência nas tomadas de decisões de empresas, governos, cidadãos, tornando as atividades urbanas mais humanizadas, eficientes e sustentáveis nas esferas econômica, social, ecológica e política.

Consequentemente, o foco hoje são projetos que visam tornar a economia, a mobilidade urbana, o meio ambiente, ambiente, os cidadãos e o governo mais inteligentes. A cidade passa a ser um organismo informacional que reage e atualiza todos sobre suas condições a qualquer hora. " (LEMOS, 2013, p.48)

\section{DESAFIOS PROPOSTOS}

O maior desafio, talvez, seja identificar de forma técnica e científica diretrizes projetuais, quantitativas e qualitativas de forma com que se possa, sob a ótica dos conceitos de Cidades Biofílicas Inteligentes, analisar e obter índices em média escala, definindo aspectos de aplicações através de uma matriz organizacional, visando um quadro de análises para estes termos.

O MIMOS (Malaysian Institute of Microeletronic Systems), por exemplo, indica que entre os usos figurados do termo "cidade inteligente" incluem-se as noções de "cidade invisível", "cidade da informação", "cidade ligada por cabos", "telecidade", "cidade baseada no conhecimento", "cidade virtual", "comunidades eletrônicas", "espaços eletrônicos", "flexibilidade", "teletopia", "cibercidade" e outras, a maioria das quais não apresenta elementos de inteligência. (KOMNINOS, 2008, p.2).

Mesmo em uma cidade convencional (não tão inteligente) podem-se aplicar alguns conceitos smart surgidos a partir da união de alguns sistemas de inovação como parques tecnológicos, bairros tecnológicos, polos de inovação. Estes clusters de atividades são dotados de aplicações de suas redes digitais na sociedade da informação e seu mérito está na habilidade de pôr em prática a relação das três formas de inteligência - humana, coletiva e artificial (KOMNINOS, 2008).

O banco de dados que compõe um SIG (Sistemas de Informações Geográficas) é uma das tecnologias mais empregadas para o planejamento e precisão das tomadas de decisões no âmbito urbano. SIG é um termo aplicado a sistemas que realizam o tratamento computacional de dados geográficos e tem ampla gama de aplicações (THOMÉ, 1998). O SIG está relacionado às atividades de sensoriamento remoto, cadastros e outros tipos de pesquisa e investigações de campo para captura de dados.

Segundo Korte (2001), o SIG é uma ferramenta utilizada para análises de informação geográfica que usa funções de dados geométricos ligados a tabelas de atributos alfanuméricos, o que permite a visualização espacial dos dados através de interfaces gráficas. 


\section{MÉTODOS DE ANÁLISE}

De acordo com Amado (2005) na década de 90 as cidades perceberam a pouca disponibilidade dos recursos naturais e começaram a pensar em maneiras de otimizar o uso e gestão dos recursos existentes, então, para apoiar esta atitude o termo de cidade inteligente surgiu. Atualmente as Smart Cities abrem caminho utilizando de seus projetos inovadores para serem desenvolvidos em larga escala, na busca de respostas frente aos possíveis efeitos da escassez de recursos naturais.

Verificado que o processo inteligente vem acontecendo de forma exponencial nas mais diversas áreas, Strapazzon (2010) diz que para uma cidade ser inteligente precisa obrigatoriamente movimentar seis condições básicas para o ser humano: a economia, sociedade, governo, mobilidade, meio ambiente e consequentemente qualidade de vida. Nesse sentido, de acordo com a Competitive European Cities (PARKINSON et al., 2004) De janeiro de 2004, existem seis aspectos chave da competitividade urbana: diversidade econômica, recursos humanos qualificados, conectividade interna e externa (física, eletrônica e cultural), capacidade estratégica para mobilizar e implementar estratégias de desenvolvimento de longo prazo, inovação de empresas e qualidade de vida social, cultural e ambiental.

Visando a sistematização de um quadro metodológico quantitativo e qualitativo para a análise e classificação específica de cidades médias, integrando e incrementando o trabalho de dois referenciais no que tange o conceito de cidade, foram adicionados novos subtópicos aos propostos por Strapazzon (2010) e Competitive European Cities (PARKINSON et al., 2004). Não se pode avaliar cidades de forma inflexível e universal, portanto os itens propostos por esses dois autores ainda não possuem toda a amplitude desejada, necessitando-se incluir subtópicos para suporte metodológico de análise com base analítica em oito grandes itens: Tecnologias, mobilidade urbana, conforto, sistemas de espaços livres, ecologia, economia, turismo e patrimônio. Essa matriz relacional consta da Figura 1. 
Figura 1: Matriz relacional dos aspectos qualitativos.

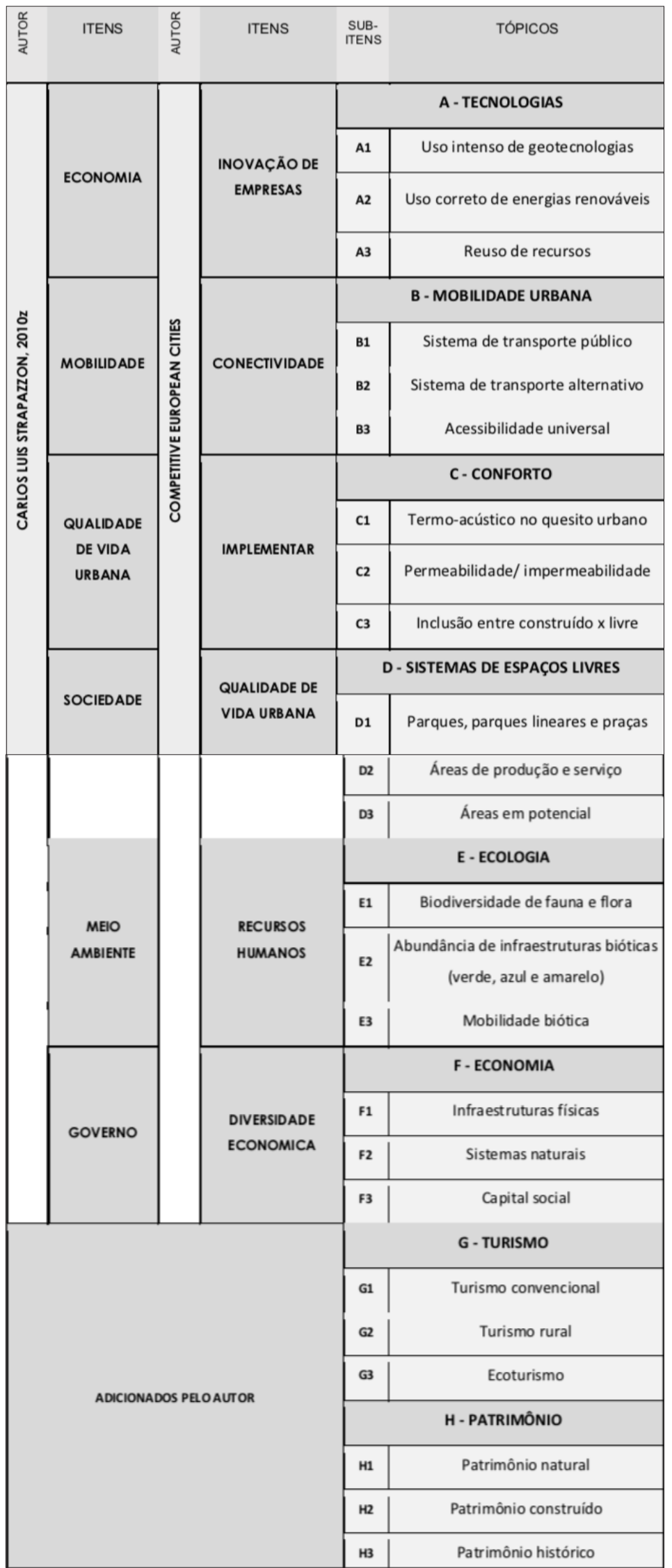

Fonte: Fonte: mod. e adapt. de Strapazzon (2010) e Parkinson et al. (2004). 


\section{TECNOLOGIAS}

Dentro do grupo Tecnologia, destacam-se o 'Uso intenso de geotecnologias - A1', o a qual se deve ao conforto do GPS nas comodidades cotidianas, seja como usuário para a prática de esportes (caminhadas, ciclismo, natação, mergulho, etc.), nos sistemas de transporte, tanto individual como coletivo, através de aplicativos para smart devices que ilustram rotas de ônibus, caminhos a se percorrer, rotas mais rápidas, condições de trânsito, ... Também se aplicam ao planejamento expansivo das cidades, facilitando a compreensão territorial. Este subitem é correlacionado com o Sistemas de Informações Geográficas (LANG; BLASCHKE, 2009).

Os subitens que seguem são ‘Uso correto de energias renováveis - A2' e 'Reuso de recursos - $\mathrm{A} 3^{\prime}$, voltando assim o pensamento para a conscientização da reutilização inteligente de recursos naturais, explorando de forma inteligente através de usinas fotovoltaicas, eólicas, biomassas, geotérmica, ósmica (...) mas que também seja aplicado na escala do usuário e da usabilidade urbana, como em postes de iluminação, pistas podotáteis geradoras de energia, carros e meios de transporte alternativos, etc.

\section{MOBILIDADE URBANA}

Para o item de mobilidade urbana, o primeiro subitem é o 'Sistema de transporte público - B1', de vital importância para a população, para que não se torne defasado, utilizando um planejamento de rotas de ônibus, com estudos de impacto. Porém deve-se também pensar em implementar trajetos ferroviários, ou até mesmo marítimos. O objetivo é que sejam funcionais à população que os utiliza.

'Sistemas de transporte alternativos - B2' são também chamados de modais alternativos e compreendem ciclovias e ciclofaixas, pistas multiuso, corredores peatonais, calçadões, ... A própria rua se enquadra neste aspecto, envolvendo a correlação entre a pista de rolagem e o passeio público, dentre outros, com análise da disponibilização e utilização ou não dos elementos, bem como sua manutenção.

A 'Acessibilidade universal - B3', no conceito geral de que a cidade precisa funcionar como um organismo vivo, sendo universal, democrática e acessível. Este quesito computa a multifuncionalidade e a caminhabilidade dos locais, a existência ou não de barreiras (naturais, antrópicas ou sociais) e as medidas adotadas para suavizá-las.

\section{CONFORTO}

Com relação ao tão necessário conforto ambiental, o primeiro subitem é ‘Termoacústico no quesito urbano - $\mathrm{C1}^{\prime}$, que faz menção à vivencia e experiência dos usuários do local, quais estratégias para amenizar o ruído das vias ou até mesmo técnicas para criação de microclimas diferenciados no decorrer do percurso urbano. 
'Permeabilidade e impermeabilidade - C2' é outro fator importante a ser analisado, referente aos materiais de pavimentação a serem utilizados de forma a contribuir para o escoamento de águas pluviais, incluindo canteiros centrais, canteiros de calçadas e até mesmo hortas comunitárias dentro de centros urbanos.

A 'Relação entre o construído x livre - C3' é muito estudada devido à sua pluralidade de formas. Este aspecto relaciona ambos subitens anteriores com a visão de potencialidades para se trabalhar os quesitos no trajeto urbano.

\section{SISTEMAS DE ESPAÇOS LIVRES}

'Parques, parques lineares e praças - D1' trazem ao debate a existência e utilização ou não destes espaços. Neste item computa-se todos os grandes espaços verdes, executados e previstos em plano de gestão e a funcionalidade dos mesmos no contexto urbano. No entanto o termo 'espaços livres' abrange também qualquer cobertura vegetal presente no meio urbano, como, por exemplo, quintais, canteiros centrais, lotes sem edificações, ... (RUMBLE et al., 2019). Estas áreas também atuam como agentes ativos para a permeabilidade do solo bem como da paisagem, possuindo grande potencial para introdução de cobertura arbórea, uma vez que seus níveis de presença sejam altos. Nas cidades brasileiras, os quintais são um tipo muito comum de uso residencial, independentemente do nível socioeconômico do bairro. Tipicamente, $30 \%$ ou mais das terras urbanas nas cidades brasileiras se apresenta na forma de quintais, cujo potencial para aumentar a cobertura de árvores é notável (ANGEOLETTO; FELLOWES; SANTOS, 2018).

'Áreas de produção e serviço - D2' são referentes aos distritos industriais que atuam de forma fundamental para o funcionamento da cidade, porém é necessário maior cuidado em disponibilizar nesses bairros o acesso e respeito ao fluxo biótico da natureza. Já 'Áreas em potencial - D3' são espaços que apresentam localização privilegiada no contexto urbano, porém possuem baixo fluxo, como indústrias abandonadas, áreas militares, setores educacionais e até mesmo terrenos baldios. Estes sítios são por vezes marginalizados e cabe a essa análise identificar sua utilização ou não, bem como a proposição para seu uso.

\section{ECOLOGIA}

Antes de qualquer tipo de urbanização ocorrer, naquele espaço existia um ecossistema e uma biodiversidade de fauna e flora característica de cada sítio. Então é importante que a cidade biofílica e inteligente entenda e respeite a existência destes seres, provendo de formas diferenciadas de crescimento urbano de maneira a compatibilizar e se conviver em harmonia e equilíbrio.

Cabe também neste item compreender as necessidades e funcionalidades de cada infraestrutura biótica, seja ela verde, azul ou amarela definindo melhores formas de abordagem para que se possa usufruir destas de forma consciente e não apenas consumi-las. Estas infraestruturas dizem respeito ao fluxo ecossistêmico individual de cada ser presente, 
desta forma exige meticulosidade de projeto, visto que as ações tomadas para a modificação destes locais irão refletir no habitat de todos os seres (seja direta ou indiretamente).

Desta forma elencaram-se os itens Biodiversidade de Fauna e Flora - E1', fazendo jus a importância da convivência e da integração dos mesmos com o espaço urbanizado (na medida do possível) através de infraestruturas e artifícios provindos do green thinking. Abundância de Infraestruturas Bióticas - Verde, Azul e Amarela - E2' são relativas à presença intensificada e impulsionada destas estruturas no meio urbano, através de corredores, dando continuidade às manchas vegetadas e possibilitando que E1' e E3' ocorram.

Mobilidade Biótica - E3' é a sumarização como resultado obtido ao se implementar E1' e E2', por possibilitar estes espaços o fluxo biótico criado neste microclima é de suma importância para o gerenciamento destas espécies bem como para conscientização ambiental dos usuários.

\section{ECONOMIA}

Para mensurar a economia aplicada a espaços públicos foram definidos os itens 'Infraestruturas físicas - F1', quantificando a existência ou não de espaços que auxiliem e modifiquem o percurso e o comércio bem como a movimentação de pessoas pelo local.

'Sistemas naturais - F2' estão presentes através da análise de sua existência e manutenção, de forma com que sejam duradouros. O 'Capital social - F3' destaca as necessidades individuais dos cidadãos, também levando em consideração o bem coletivo, as áreas de necessidade básica como institucional, saúde, relação entre unidades vizinhas, centros comunitários, dentre outros.

\section{TURISMO}

O turismo é dividido entre três subitens, o 'Turismo convencional - G1', que é aquele vinculado aos centros e edificações turísticas, incluindo o turismo religioso, o de negócios, o litorâneo, dentre outros. Em contraponto o 'Turismo rural - G2' abrange fazendas, retiros, acampamentos, áreas de pesca esportiva, hipismo, entre outros.

Em terceiro, o 'Ecoturismo - G3' apresenta-se como uma alternativa viável, sendo o mais próximo possível da natureza e da vida selvagem. Este enfatiza o conceito de biofilia, apresentando várias opções de entretenimento como visita a grutas e cavernas, mergulhos e flutuações, observação de pássaros, observação astronômica, caminhadas, trilhas, mountain bike, safaris fotográficos, dentre outros.

\section{PATRIMÔNIO}

O 'Patrimônio natural - H1' diz respeito ao que usufruímos da natureza, e não apenas ao que é contemplado, como no G2 e G3. 
O 'Patrimônio construído - H2', que pode ser classificado como antrópico, pois envolve tudo o que foi construído e adaptado pelo homem. Findando este tópico, 'Patrimônio histórico - H3' faz menção a riqueza histórica e memória da cidade, se a mesma é contada a partir da paisagem visual ou se é ignorada.

\section{CONSIDERAÇÕES FINAIS}

Para se ter plena concepção do quadro urbano atual são, idealmente, necessários métodos exequíveis para mensuração através de quantificação e qualificação das cidades, elencados entre níveis de inteligência biofílica.

O objetivo da matriz relacional desenvolvida é categorizar cada item, porém percebese a correlação quase simbiótica entre eles, sobrepondo-se e interligando os assuntos, pois pertencem todos ao mesmo meio, como espaços de circulação, espaços livres, meio antrópico e patrimônio natural, vinculados igualmente à ecologia.

Desta forma deve-se ter em mente que a análise é holística e a matriz de critérios tende a aumentar conforme surgem aspectos modificadores da qualidade de vida urbana, num constante ciclo retroalimentado.

\section{REFERÊNCIAS}

ADENE - Agência para a Energia. Guia da eficiência energética. Algés, 2010. Disponível em https:// ploran.com/artigos/guia_eficiencia_energetica_adene.pdf Acesso em: 10 out. 2018.

AMADO, Miguel et al. Oeiras E-City Modelo de Cidade Energeticamente Eficiente. Lisboa: Caleidoscópio, 2014. v. 1.

AMADO, Miguel. The operative process in Sustainable Urban Planning. In: KUNGOLOS, A.G.; BREBBIA, C.A.; BERIATOS, E. (Eds). Sustainable Development and Planning II. Southampton: WIT Press, 2005. v.1. p. 181-191.

ANDRADE, Thompson A.;SERRA, Rodrigo V. Cidades Médias Brasileiras. Brasília: IPEA, 2001. Disponível em http:/ / repositorio.ipea.gov.br/handle/11058/3081 Acesso em: 13 mar. 2019.

ANGEOLETTO, Fabio; FELLOWES, Mark D.; SANTOS, Jeater W.M.C. Counting Brazil's Urban Trees Will Help Make Brazil's Urban Trees Count. Journal of Forestry, v. 116, n. 5, p. 489-490, 2018.

BEATLEY, Timothy. Biophilic Cities: integrating nature into urban design and planning. Washington, DC: Island Press, 2010.

BLOOMBERG, Laura; CROSBY, Barbara C.; BRYSON, John M. Public Value Governance: Moving Beyond Traditional Public Administration and the New Public Management. Public. Admin. Review, v. 74, p. 445-456. Disponível em: <https://onlinelibrary.wiley.com/doi/full/10.1111/puar.12238> Acesso em: 13 abr. 2019.

CURY, Mauro J.F.; MARQUES, Josiel A.L.F. A Cidade Inteligente: uma reterritorialização. Redes, v. 22, n. 1, p. 102-117, 2016. doi.org/10.17058/redes.v22i1.8476.

GIL-GARCIA, J. Ramon et al. A Comprehensive View of the 21st Century City: Smartness as Technologies and Innovation in Urban Contexts. In: GIL-GARCIA, J. Ramon; PARDO, Theresa A.; NAM, Taewoo. Smarter as the New Urban Agenda: A Comprehensive View of the 21st Century City. New York: Springer, 2016. p. 1-19. 
HERZOG, Cecilia P. Cidades para todos: (re) aprendendo a conviver com a natureza. Rio de Janeiro: Mauad X/ Inverde, 2013.

KOMNINOS, Nico. Cidades Inteligentes - Sistemas de inovação e tecnologias da informação ao serviço do desenvolvimento das cidades. 2008. Disponível em: < https://www.urenio.org/wp-content/ uploads/2008/11/cidades-inteligentes.pdf> Acesso em: 09 out. 2019.

KORTE, George B. The GIS Book: how to implement, manage, and assess the value of Geographic Information Systems. 5.ed. New York: OnWorld Press, 2001. Disponível em: <https://books.google.com. br/books?id=_C6oPvJ5S_EC\&printsec=frontcover\&hl=pt-BR\&source=gbs_ge_summary_r\&cad=0\#v=on epage\&q\&f=false $>$ Acesso em: 12 jan. 2018

LANG, S.; BLASCHKE, T. Análise da Paisagem com SIG. São Paulo: Oficina de Textos, 2009.

LEMOS, André. Cidades inteligentes: de que forma as novas tecnologias - como a computação em nuvem, o big data e a internet das coisas podem melhorar a condição de vida nos espaços urbanos? Gvexecutivo, v.12, 2, [s.p.], 2013. Disponível em: http://bibliotecadigital.fgv.br/ojs/index.php/gvexecutivo/article/ viewFile/20720/19454 Acesso em: 5 out. 2019

NAM, Taewoo; PARDO, Theresa A. Conceptualizing smart city with dimensions of technology, people, and institutions. In: ANNUAL INTERNATIONAL DIGITAL GOVERNMENT RESEARCH CONFERENCE. 12, 2011, College Park. Anais ... p. 282-291. Disponível em: https:/ /www.researchgate. net/publication/221585167_Conceptualizing_smart_city_with_dimensions_of_technology_people_and_ institutions Acesso em: 9 out. 2019.

PARKINSON, Michael; HUTCHINS, M.; SIMMIE, James et al. Competitive European Cities: Where do the Core Cities Stand? London: ODPM, 2004.

PELLEGRINO, Paulo; MOURA, Newton B. Estratégias para uma infraestrutura verde. São Paulo: Manole, 2017.

PONTES, Beatriz M.S. As cidades médias brasileiras: os desafios e a complexidade do seu papel na organização do espaço regional (década de 1970). In: SPÓSITO, Maria E.B. Urbanização e cidades: perspectivas geográficas. Presidente Prudente: [s.n.], 2001. p.569-607.

ROSSETTO, A.M. Proposta de um sistema integrado de gestão do ambiente urbano (Sigau) para o desenvolvimento sustentável de cidades. Florianópolis, 2003. 423 f. Tese (Doutorado em Engenharia de Produção) - Universidade Federal de Santa Catarina, UFSC.

RUMBLE, Heather; ANGEOLETTO, Fabio; CONNOP, Stuart et al. Understanding and applying ecological principles in cities. In: LEMES de OLIVEIRA, Francisco; MELL, Ian (Eds.). Planning Cities with Nature: Theories, Strategies and Methods. Amsterdam: Springer Nature, 2019.

SILVEIRA, Rogério L.L. Redes e Território: uma breve contribuição geográfica ao debate sobre a relação sociedade e tecnologia. Biblio 3w, Barcelona, v. 8, n. 451, p. 1-20, 2003. Disponível em: http://www. ub.edu/geocrit/b3w-451.htm Acesso em: 2 out. 2019.

SPÓSITO, Maria E.B.; SILVA, William R. Perspetivas da urbanização: reestruturação urbana das cidades. Rio de Janeiro: Consequência, 2017.

STRAPAZZON, Carlos L. Smart Cities. In: ROVER, Aires J.; GALINDO, Fernando. O governo eletrônico e suas múltiplas facetas. Zaragoza: Prensas Universitárias de Zaragoza, 2010. p. 263-282.

THOMÉ, Joseph. Interoperabilidade em Geoprocessamento: conversão entre Modelos Conceituais de Sistemas de Informação Geográfica e comparação com o Padrão Open Gis. São José dos Campos: INPE, 1998.

ZOMER, Lara; MOUSTAID, Elhabib; MEIJER, Sebastiaan. A meta-model for including social behavior and data into smart city management simulations. In: WINTER SIMULATION CONFERENCE. 1, 2015, Huntington Beach. Proceedings... p. 1705 - 1716. 2015.

Data de submissão: 15/ mar. / 2019

Data de aceite: 14/ ago. / 2019 